Lepr Rev (1983) 54, Special Issue, 23S-30S.

\title{
Recent studies of antileprosy drugs
}

\author{
C C SHEPARD, ROSALIND M VAN LANDINGHAM \\ AND LAURA L WALKER \\ Department of Health and Human Services, Public Health Service, \\ Centers for Disease Control, Center for Infectious Diseases, Atlanta, \\ Georgia 30333
}

Summary As a part of the programme of the Therapy of Leprosy (THELEP) Scientific Working Group, a number of compounds with potential activity against Mycobacterium leprae were prepared in other laboratories. We report here the results of studies of their activity against $M$. leprae with the use of the kinetic method in mice. A modified protocol is described that facilitates comparison of drugs in the same experiment. Two analogues of cycloserine, glycylhydroxamic acid and beta-analylhydroxamic acid were inactive in a dosage of $0.1 \%$ in the diet. Isoetam, (D-2,2'-(ethylendiimino)-di-l-butanol)di-isoniazid methane sulphonate was also inactive at this dosage. Three compounds related to dapsone, 4-nitro- $\mathrm{N}^{\prime}$-phenylsulphonamide, 4-amino- $\mathrm{N}^{\prime}$-phenylsulphonamide, and 4,4'-diaminobenzene sulphonic acid phenyl ester, had little or no activity at dosages of $0.01 \%$ in the diet in experiments with strains shown to have normal susceptibility to dapsone. Two thiosemicarbazones, pyridinal-4-thiosemicarbazone and pyridinal-2-thiosemicarbazone, were inactive in dosages of $0.01 \%$; the latter was inactive at $0.01 \%$ in an experiment where thiacetazone was shown to have bactericidal-type activity at a dosage of $0.1 \%$ and marginal activity at $0.01 \%$. Brodimoprim, a dihydrofolate reductase inhibitor, which is related to trimethoprim but has a longer half-life, was inactive in a dosage of $0.1 \%$; it had no synergistic effect with $0.01 \%$ dapsone against a dapsone-susceptible strain. It was also inactive against a dapsone-resistant strain, alone or in combination with dapsone. The cyanimino analogs of ethionamide and prothionamide were inactive in a dosage of $0.1 \%$ against an ethionamide-susceptible strain. Experiments with a series of compounds related to chaulmoogric acid were unsuccessful because the compounds were too toxic. Experiments with a series of compounds related to clofazimine were unsuccessful because their pharmacokinetics were unfavourable for study at dosages where clofazimine itself was active. The limitations imposed by the mouse-foot-pad system are discussed and related to those in other experimental systems. 


\section{Introduction}

In collaboration with the Therapy of Leprosy (THELEP) Scientific Working Group, we have examined a number of new drugs for activity against Mycobacterium leprae in mice in the last several years. The drugs were designed and synthesized by the members of the Scientific Working Group named below. The meetings of the Scientific Working Group were a vital part of the development of these new drugs, in that they provided a focus for scientific attention and a forum for discussion that extended far beyond any one individual's competence, from drug design and synthesis to pharmacokinetics, microbiology and application to leprosy field situations. Some of the results of drug testing were published elsewhere in a collaborative study (Shepard et al., in preparation). We present here the remainder of the results to date. Although little activity was observed with the new drugs, the results can be helpful to others interested in the design of new antileprosy drugs.

\section{Materials and methods}

We used a recently described modification of the kinetic method ${ }^{1}$ and slightly modified it further (see below). In brief, CFW female mice, 6-12 weeks old, were infected in a rear foot pad with $5 \times 10^{3} M$. leprae of a 'fast' strain $^{2}$ in mouse passage. The strains used were isolated from untreated patients before 1965, that is, before the recent increase in primary dapsone (DDS) resistance in several parts of the world, and most had already been shown to be susceptible to $0.0001 \%$ DDS in the diet. The growth curve of $M$. leprae in the various groups was monitored by harvests of the infected foot pad tissues and microscopic counts of acid-fast bacteria (AFB) in the tissue suspensions. Each count was carried out on a pool of four mice per group, and there were four (untreated) control groups. The harvests were carried out at approximately 28-day intervals during the logarithmic growth phase of the controls (roughly 98-182 days, when the average count rose from $10^{4 \cdot 3}$ to $10^{6 \cdot 0} \mathrm{AFB} / \mathrm{mouse}$ ), and at 56-day intervals thereafter. Harvests were discontinued in treated groups when they reached plateau levels (usually $10^{6.0}$ to $\left.10^{6 \cdot 2} \mathrm{AFB} / \mathrm{mouse}\right)$.

The statistical significance of the difference between groups was estimated as described $^{1}$ or by a slight modification. In the described procedure, comparisons between a treated group and the control groups are taken care of automatically by the protocol, and in general growth delays as little as 25 days are detectable $(P<0.05)$. Based on a generation time of 12.5 days, a value that fits our results well, 25 days of growth delay corresponds to a loss in numbers of viable $M$. leprae of $75 \%$. A disadvantage is that when comparisons of treated groups are important, adjustment of the protocol is usually needed to increase the number of counts from the particular treated groups. This adjustment has proved difficult to 
accomplish sometimes while maintaining the 28-day counting interval and carrying out other laboratory activities. Consequently, when comparisons of treated groups are important, a modified protocol has been used in which counts are carried out on four individual mice from each group. The significance of the differences between groups at a particular time is estimated by the two-sample rank test (Mann-Whitney test) with the use of tables giving the exact probability value, for example Table $\mathrm{J}$ in Siegel. ${ }^{3}$ The period selected for comparison is that when one, or both, of the groups being compared are in the logarithmic phase. As in previous studies ${ }^{1}$ the $P$ values from each successive period are multiplied to give the final $P$ value for the differences between groups.

\section{Results}

GLYCYLHYDROXAMIC ACID AND BETA-ANALYLHYDROXAMIC ACID

These compounds, which are sterically related to cycloserine, were prepared by J B Hines, University of South Carolina. Their antimycobacterial properties have been described. ${ }^{4,5}$ At a dosage of $0 \cdot 1 \%$ in the diet, they were inactive against $M$. leprae (Table 1).

\section{ISOET AM}

D-2,2'-(ethylendiimino)-di-l-butanol)-di-isoniazid methane sulphonate, is a drug that has been used in the treatment of patients with tuberculosis. It was received from J Colome and J Castillo, Ferrer Internacional, Barcelona, Spain. At a dosage of $0 \cdot 1 \%$ in the diet, it was not active against $M$. leprae (Table 1 ).

\section{SULPHONE-RELATED COMPOUNDS}

Three compounds (4-nitro- $\mathrm{N}^{\prime}$-phenylsulphonamide or SAL1, 4-amino- $\mathrm{N}^{\prime}$ phenylsulphonamide or SAL2, and 4,4'-diaminobenzene sulphonic acid phenyl ester or SAL3) were prepared by J K Seydel, Forschungsinstitutborstel, Borstel, West Germany. ${ }^{6} \mathrm{He}$ had found them active in vitro against certain cultivable mycobacteria at about the same levels as DDS. Accordingly, in experiment M1-23-79 we tested SAL1 and SAL2 against $M$. leprae at dosages near the minimum effective dosage (MED) of DDS. DDS, at $0.0001 \%$ in the diet, had the expected activity, but SAL1 and SAL2 were inactive even at $0.001 \%$ in the diet. In a second experiment, M6-30-80B, the drugs were tested at $0.01 \%$, a dosage of DDS that produces levels of DDS in the plasma and tissues that are about the same as those produced in man by standard daily dosages. SAL2 had a minimal amount of activity with an estimated 29 days of growth delay, But SAL1 and SAL3 were inactive. DDS again had the expected amount of activity; it 
Table 1. Results of studies of several compounds for their activity against $M$. leprae by the kinetic method $^{\mathrm{a}}$.

\begin{tabular}{|c|c|c|c|c|}
\hline Experiment and drug & Dosage $(\%)$ & $\begin{array}{l}\text { Time given } \\
\text { (days) }\end{array}$ & $\begin{array}{l}\text { Growth delay } \\
\text { (days) }\end{array}$ & $\begin{array}{l}\text { Probability } \\
\text { value }^{b}\end{array}$ \\
\hline \multicolumn{5}{|l|}{$\mathrm{M} 10-30-78^{\mathrm{c}}$} \\
\hline GHA & $0 \cdot 1$ & $70-126$ & 0 & \\
\hline BHA & $0 \cdot 1$ & $70-126$ & 0 & \\
\hline Isoetam & $0 \cdot 1$ & $70-126$ & 0 & \\
\hline \multicolumn{5}{|l|}{ M 1-23-79c } \\
\hline SAL1 & $0 \cdot 001$ & $70-126$ & 0 & \\
\hline , & $0 \cdot 0001$ & $70-126$ & 0 & \\
\hline, & $0 \cdot 00001$ & $70-126$ & 0 & \\
\hline SAL2 & 0.001 & $70-126$ & 8 & NS \\
\hline, & 0.0001 & $70-126$ & 4 & NS \\
\hline, & $0 \cdot 00001$ & $70-126$ & 0 & \\
\hline DDS & 0.0001 & $70-126$ & 74 & $<0.02$ \\
\hline & $0 \cdot 00001$ & $70-126$ & 14 & NS \\
\hline TSCL1 & $0 \cdot 01$ & $70-126$ & 28 & $<0.06$ \\
\hline TB1 & $0 \cdot 01$ & $70-126$ & 0 & \\
\hline \multicolumn{5}{|l|}{ M 6-30-80 } \\
\hline DDS & 0.01 & $70-126$ & $>114$ & $<0.0016$ \\
\hline SAL1 & 0.01 & $70-126$ & 26 & NS \\
\hline SAL2 & 0.01 & $70-126$ & 29 & $<0.008$ \\
\hline SAL3 & 0.01 & $70-126$ & 4 & NS \\
\hline TSCL2 & 0.01 & $70-126$ & 0 & \\
\hline TSCL2 & $0 \cdot 1$ & $70-126$ & 0 & \\
\hline TB1 & $0 \cdot 01$ & $70-126$ & 17 & $<0.04$ \\
\hline, & $0 \cdot 1$ & $70-126$ & $>100$ & $>0.0016$ \\
\hline \multicolumn{5}{|l|}{$M 11-18-81^{d}$} \\
\hline DDS & 0.01 & $70-98$ & 87 & $<0.001$ \\
\hline BDP & $0 \cdot 1$ & $70-98$ & 18 & $<0.017$ \\
\hline $\operatorname{DDS}(0 \cdot 01 \%)+\operatorname{BPP}(0 \cdot 1 \%)$ & & $70-98$ & 54 & $<0.002$ \\
\hline \multicolumn{5}{|l|}{ M $2-10-82^{d}$} \\
\hline ETH & $0 \cdot 1$ & $70-126$ & $>56$ & $<0.024$ \\
\hline DPS28 & $0 \cdot 1$ & $70-126$ & 5 & NS \\
\hline DPS29 & $0 \cdot 1$ & $70-126$ & 0 & NS \\
\hline \multicolumn{5}{|l|}{ M 5-21-82d,e } \\
\hline DDS & 0.01 & 70--98 & 14 & NS \\
\hline $\mathrm{BDP}$ & $0 \cdot 1$ & $70-98$ & 6 & NS \\
\hline $\operatorname{DDS}(0 \cdot 01 \%)+\operatorname{BDP}(0 \cdot 1 \%)$ & & $70-98$ & 15 & NS \\
\hline
\end{tabular}

a Abbreviations: GHSA, glycylhydroxamic acid; BHA, beta-analylhydroxamic acid; SAL1, 4-nitro- $\mathrm{N}^{\prime}$-phenylsulphonamide; SAL2, 4-amino- $\mathrm{N}^{\prime}$-phenyl-sulphonamide; DDS, dapsone; TSCL1, pyridinal-4-thiosemicarbazone; TB1, thiacetazone; SAL3, 4,4'-diaminobenzenesulphonic acid phenyl ester; TSCL2, pyridinal-2-thiosemicarbazone; BDP, brodimoprim; ETH, ethionamide; DPS28, cyanimino analog of prothionamide; DPS29, cyanimino analog of ethionamide.

${ }^{\mathrm{b}}$ For difference from untreated control.

${ }^{\mathrm{c}}$ Protocol as described in 1.

${ }^{\mathrm{d}}$ Modified protocol. See text.

${ }^{\mathrm{e}}$ Tested against a dapsone-resistant strain of $M$. leprae. 
manifested bactericidal-type activity with $>114$ days of growth delay, well in excess of the 56 days of drug administration. (Pure bacteriostasis contemporaneous with the period of drug administration would have produced 56 days of growth delay.)

\section{THIOSEMICARBAZONES}

Two compounds, pyridinal-4-semicarbazone (TSCL1) and pyridinal-2-semicarbazone (TSCL2), were received from Dr Seydel. In these compounds the acetylaminobenzene portion is replaced by a pyridine group. TSCL1 had questionable activity in one experiment at a dosage of $0.01 \%$, and TSCL2 had no activity in another experiment at dosages of $0.01 \%$ and $0.1 \%$. In the former experiment thiacetazone (TB1) was inactive at $0.01 \%$; in the latter, 17 days of growth delay was observed with a dosage of $0 \cdot 01 \%$ and $>100$ days with $0 \cdot 1 \%$. By the continuous method of drug administration, TB1 has previously been found to be partially active in a dosage of $0.01 \%$ against most strains of $M$. leprae and completely active in a dosage of $0 \cdot 1 \%{ }^{7} \cdot{ }^{7,8}$

\section{BRODIMOPRIM}

This drug, a 2,4-diaminopyrimidine, is a dihydrofolate reductase inhibitor. For some years now, one has hoped to find a dihydrofolate reductase inhibitor that will be highly active in combination with dapsone, as a result of sequential blockade in the folate biosynthetic pathway. It differs from trimethoprim in that the central methoxy group is replaced by a bromine, and it has a longer half life. Trimethoprim, in a dosage of $0.1 \%$, has previously been found to be inactive against $M$. leprae when administered alone, and it had no additive effect when administered with dapsone. ${ }^{7}$ In our studies, $0 \cdot 1 \%$ brodimoprim was inactive alone, and it did not increase the effect of dapsone (Table 1). Against a DDS-resistant strain it had no effect alone or in combination with dapsone (experiment M 5-21-82).

\section{CYANIMINO ANALOGS OF PROTHIONAMIDE AND ETHIONAMIDE}

Study of these compounds was suggested by Michael Tute, Pfizer Central Research, Sandwich, Kent, United Kingdom, and the compounds were synthesized by M Hooper and D P Self, Sunderland Polytechnic, Sunderland, United Kingdom (under a WHO project grant 790490). The precedent was the replacement of the thione group of metiamide by a cyanoimino group to yield cimetidine, a potent $\mathrm{H}_{2}$ antagonist with low incidence of side-effects. Thus, the $-\mathrm{CSNH}_{2}$ group of prothionamide and ethionamide was replaced with a $-\mathrm{CNCNNH}_{2}$ group. DPS 28 and DPS 29, however, were found inactive in a concentration of $100 \mu \mathrm{g} / \mathrm{ml}$ against a strain of $M$. tuberculosis that was susceptible 
to $1.2 \mu \mathrm{g}$ prothionamide $/ \mathrm{ml}$ and $0.6 \mu \mathrm{g}$ ethionamide $/ \mathrm{ml}$. Table 1 shows that the compounds were also inactive against $M$. leprae.

\section{OTHER COMPOUNDS}

In this same period, three inconclusive experiments were also carried out (results not shown). In one, a series of new clofazimine-related compounds were tested along with clofazimine controls. Although the clofazimine controls had the expected growth delay, the new compounds were inactive. Although demonstrating good activity in vitro the new compounds had basic substitutions that increased their solubilities and altered their distributions in the body, as evidenced by lack of pigmentation of the fatty tissues. Since the compounds were all administered in dosages of $0.001 \%$ and $0.0001 \%$, that is, at dosages near the MED of clofazimine, the inactivity of the new compounds could have arisen from altered pharmacokinetics.

Two inconclusive experiments were carried out with chaulmoogric acid and several related compounds. The compounds were administered in propylene glycol by injection. In one experiment they were given intraperitoneally 5 days a week in a dosage of $0.6 \mathrm{mg}$, but this proved too toxic, and after 15 days, the dose was lowered to $0.2 \mathrm{mg}$. Deaths continued, however, and the dead mice had a knot of intestine that was tied together with peritoneal adhesions. Two few mice remained alive to allow reliable conclusions. In the other experiment the drugs were given subcutaneously in a dose of $2 \mathrm{mg}$ three times a week. The mice developed subcutaneous lesions and many died. In both experiments only the mice receiving the vehicle remained well.

\section{Discussion}

Now, after 23 years of use, the mouse foot pad remains the most convenient and reliable laboratory system for the study of drugs for activity against $M$. leprae. Certain strains of rats have been proposed rather than mice on the basis that their metabolic disposition of dapsone is more similar to that of man, ${ }^{3}$ but this feature would be helpful only for particular purposes. Nine-banded armadillos have been proposed on the basis that they develop a disease similar to that of human lepromatous disease and that their uniovular quadruplets provide a set of genetically uniform animals. The mouse is much the more convenient, however, especially in view of the fact that the statistical requirement for numbers of animals per group does not decrease when the experimental animal is large or inconvenient to work with. The mechanism of action of nearly all antimicrobials is against some particular metabolic feature that is characteristic of the microorganisms and not the host. Fortunately, the mouse foot pad provides an environment in which the $M$. leprae can be exposed to a drug during the equivalent of at least six generations (75 days) of logarithmic bacillary growth in 
the controls. In this sense, at the present the mouse is providing the most convenient substitute for a favourable artificial medium. Of course, genetic homogeneity can be provided by inbred lines of mice in very large numbers.

Compared with potential in vitro systems, however, the disadvantages in the use of the mouse are distinct. It is true that analogs of a compound known to be active in low dosages can be compared with the known compound at its minimal effective dosage. Thus it is possible to test analogs of dapsone or rifampicin when only $10-20 \mathrm{mg}$ is available if the pharmacokinetics of the analog is similar to that of the parent compound. Nevertheless, the first screening of a new compound usually requires $10-15 \mathrm{~g}$ of drug (usually the maximum tolerated dosage, of ten about $0.1 \%$ in the diet or $10 \mathrm{mg} /$ day by injection, is administered for about 2 months). With many antimicrobials, these dosages provide plasma and tissue concentrations of $10-30 \mu \mathrm{g} / \mathrm{ml}$. Thus in vitro procedures would be needed to screen entirely new drugs at this level if they are available in only milligram amounts. Unfortunately, reliable methods for the cultivation of $M$. leprae in vitro through even one generation of growth do not appear to be available yet.

Other disadvantages of an in vivo model were displayed in this work. The drug to be tested may be too toxic or the pharmacokinetics (absorption, distribution, biotransformation, excretion) may be unfavourable. It might be argued that these unsuitable properties of a compound would prevent its use in man anyhow. Toxicity and unsuitable pharmacokinetics, however, may be a function of a part of the molecule that is distinct from that responsible for its antibacterial activity, and it may be possible to design active congeners with more suitable properties if an adequate degree of antibacterial activity is present.

In the case of the compounds related to clofazimine that we tested, the altered pharmacokinetics were revealed rather simply by their failure to pigment the body fat. Changes had been expected because the compounds had a basic substitution and greater solubility in water. In the case of the new compounds listed in Table 1, they were analogs of known compounds, and it is unlikely that they had unsuitable pharmacokinetics. If necessary, firm evidence that the compound is reaching the site of the microorganism can be provided by the development and application of a procedure for the specific determination of the active compound in body fluids, in a manner similar to that used for the study of thioamides against $M$. leprae in mice (Shepard et al, in preparation).

When one is studying drugs that have already been shown to be active in mice against other infectious agents, toxicity and unsuitable pharmacokinetics are much less likely to interfere. For example, in a current experiment we are studying a series of beta-lactam antibiotics; they have already been shown to be active in animals and man against other bacteria.

\section{Acknowledgement}

This study received support from the THELEP component of the UNDP/World Bank/WHO Special Program for Research and Training in Tropical Diseases. 


\section{References}

${ }^{1}$ Shepard CC. Statistical analysis of results obtained by two methods for testing drug activity against Mycobacterium leprae. Int J Lepr, 1982; 50: 96-101.

2 Shepard CC, McRae DH. Hereditary characteristic that varies among isolates of Mycobacterium leprae. Infect. Immun, 1971; 3: 121-126.

3 Siegel S. Nonparametric Statistics for the Behavioral Sciences. McGraw Hill, 1956.

${ }^{4}$ Gale GR, Hawkins JE. Antimycobacterial properties of glycly hydroxamic acid and beta-analyl hydroxamic acid. Am Rev Respir Dis, 1965; 92: 642-646.

${ }^{5}$ Gale GR, Hynes JB. Further studies of the antimycobacterial agents glycyl hydroxamic acid and $\beta$-analyl hydroxamic acid. Can J Microbiol, 1966; 12: 73-81.

${ }^{6}$ Seydel JK. Prediction of the in vitro activity of sulfonamides synthesized from simple amines by the use of electronic data obtained from the simple amines. J Med Chem, 1971; 14: 724-729.

7 Shepard CC. A survey of the drugs with activity against M. leprae in mice. Int J Lep, 1971; 39: 340-348.

${ }^{8}$ Colston MJ, Hilson GRF, Ellard GA, Gammon PT, Rees RJW. The activity of thiacetazone, thiambutosine, thiocarlide, and sulphamethoxypyridazine against Mycobacterium leprae in mice. Lepr Rev, 1978; 49: 101-113.

9 Gordon GR, Ghoul DC, Murray JF, Jr, Peters JH, Levy L. Tissue levels of dapsone and monoacetyldapsone in rats and mice following dapsone. Int J Lepr, 1974; 42: 373-374. 